\title{
Sentiment Analysis on Evacuation and Relief Operation in the Philippines
}

\author{
Maria Charmy A. Arispe ${ }^{1}$, Rosemarie T. Bigueras ${ }^{2}$, Jocelyn O. Torio ${ }^{3}$, Daniel E. Maligat, Jr. ${ }^{4}$ \\ ${ }^{1}$ Bicol University Polangui Campus, Polangui, Albay, Philippines, cham.arispe@ gmail.com \\ ${ }^{2}$ Camarines Norte State College, Daet, Camarines Norte, Philippines, rosemariebigueras@ gmail.com \\ ${ }^{3}$ Camarines Norte State College, Daet, Camarines Norte, Philippines, jocelynt77@ gmail.com \\ ${ }^{4}$ Camarines Norte State College, Daet, Camarines Norte, Philippines, dmj234@ yahoo.com
}

\begin{abstract}
Individual sentiments on current certain issues were expressed through social media as well as to get the pulse of the majority population in certain circumstances, specifically on disaster management issues. To conduct a sentiment analysis of the disaster evacuation and relief operations in the Philippines, the researchers used Twitter social media. The qualitative research methods and sentiment analysis were used to collect and analyze the data. The GetOldTweets application was used to collect the tweets related to disaster relief and evacuation from the year 2013 to the year 2017 and serves as the data sets for the analysis of sentiments. Social media posts written in English and Filipino tweets were analyzed by using the Waikato Environment for Knowledge Analysis (WEKA) tools to manually annotate positive or negative tweets and validate classification model output using stratified cross validation and support vector machines (SVMs) algorithms. The manual classification model received a high percentage of correctly categorized instances using the 10-fold cross validation with the classifier SVMs. Tweets with positive and negative sentiments will significantly improve to have an effective and efficient activity for relief and evacuation. Government needs several measures to strengthen the evacuation process and the management system for relief operations.
\end{abstract}

Key words : disaster, evacuation, machine learning, relief operations, sentiment analysis, vector machine.

\section{INTRODUCTION}

Evacuation and relief operations are the main disaster response activity during a disaster. Consequently, emergency management and successful operations depend heavily on the cooperation of various organizations. It will lessen the impact of the disaster on human life as well as assist the person affected during the disaster in their basic needs [1]. A significant number of homeless people have to be evacuated from the emergency sites to temporary shelters after an emergency. The evacuation power, however, is insufficient, evacuees have to be evacuated in batches and even evacuated to evacuation centers, they still have to wait for aid because the relief services are insufficient [2].

It is critical for the organizations concerned to consider the rapid change in the human population within impact areas and recognize the social perception of local residents when designing evacuation plans. [3]. And, despite the good governance of the organization concerned in the process of disaster relief and evacuation, there are some sentiments that can read about the evacuation and relief service management system in social media. Information coming from social media can help individuals to be aware of what is happening in the world, in particular about disaster. Thus, individual and affected population of various disasters in different locations in the Philippines have different sentiment about the country's evacuation and relief operations management program.

Social perception means opinions or sentiments. Twitter is now one of the social media that is used in many cases to convey individual opinions or sentiments. Such opinions or sentiments may be positive, negative, or neutral. Social networking is a rich data base for evaluating the social effects of dangerous systems and human actions in crisis situations [4]. Similarly, social media offers a plethora of information during the disaster that includes information about the nature of the disaster, the emotions of people affected, and relief efforts [5].

Many researchers have conducted analyzes of sentiment in the past few years. The area of research that analyzes people's thoughts, feelings, perceptions, behaviors, and emotions towards goods, services, organizations, individuals, problems, events, subjects, and their attributes [6] and one of the forms of natural language processing to track public opinion on a specific product or topic [7] is an examination of sentiments.. Sentiment analysis is very useful nowadays in various fields of study since analyzing of sentiments in one case can be used by several individuals to solve any issue.

One of the ways now to identified or classified the sentiment of the individual in certain situations or issues is to conduct 
study in the sentiment analysis in different issues or situation. The sentiment detection methods were derived from those used for documents, but explored necessary changes to the methods for the unique characteristics of Twitter microblogs, such as message shortness and emoticon usage, etc. It can be used as an effective tool for educating humanitarian efforts and improving how insightful announcements are prepared for the people towards crises or disasters [8]. Microblogging sites share their thoughts with millions of people every day, including Twitter, where users share comments and thoughts on all in real-time [9].

Using sentiment analysis, the Filipino opinions and sentiments are conveyed and then discussed [10]. In this context, the researchers come up with this study to identify the sentiments of a Filipino in terms of evacuation and relief operation management system of the Philippines. In particular, it aims at answering the following questions: (1) what data sets may be collected for sentiment analysis on evacuation and relief operations related tweets; and (2) what are the common sentiments of the Filipino in evacuation and relief operation management system in the Philippines during the disaster?

\section{METHODOLOGY}

The researchers use qualitative research approach to analyze the data required for the evacuation and relief activity management system sentiment analysis. This study's methodology structure consists of data selection, manual tweet classification into relief and evacuation activity, data cleaning, data annotation and training set performance assessment as shown in Figure 1..

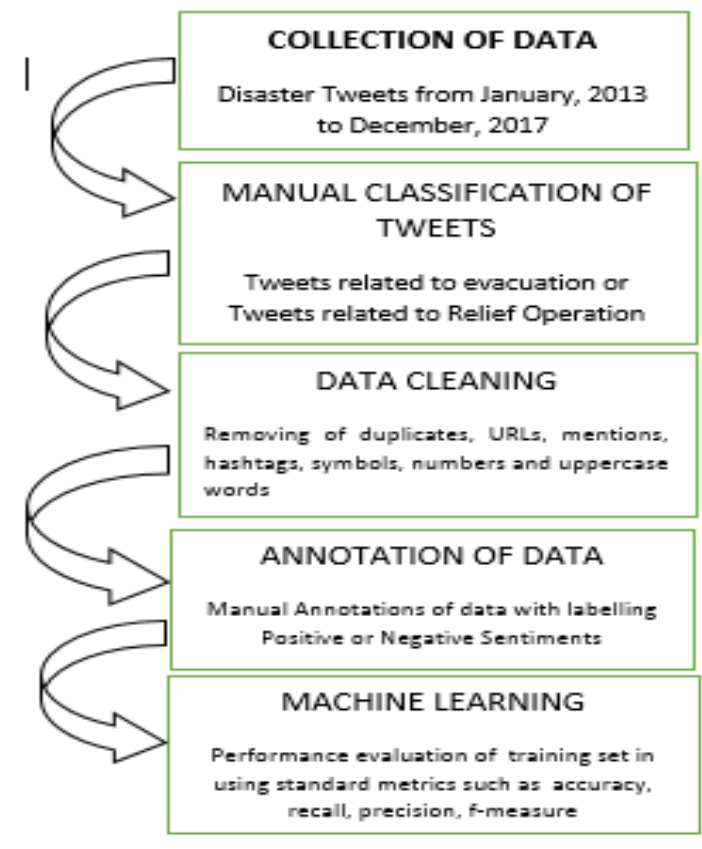

Figure 1: Methodology of the Study

Data collection is the process of getting sentiment tweets from Twitter using GetOldTweets [11]; manual classification is the process where the researchers manually classified the collected tweets into relief or evacuation process; data cleaning is removal of unnecessary words or elements of tweets such as hashtags and etc.; annotation of data is the manual labelling of the sentiments tweets into positive and negative sentiments; and machine learning is the validation of the performance of the data model.

\subsection{Data Collection and Data Source}

The researchers use GetOldTweet application programming interface, one of the application interfaces in python to collect the tweets from the twitter's raw data. The data sources are tweets about the Philippines disaster evacuation and relief activity from January 2013 to December 2017. It started in 2013, since this year's typhoon Yolanda happened, one of Philippines' biggest disasters. Many Filipinos use twitter to express their views on the disaster situation where a total of 25,123 sentiment tweets related to disaster evacuation and relief operations are contained in the data sample collected by the researchers.

\subsection{Manual Classification}

Out of 25,123 tweets, the researchers manually classified 13,137 tweets related to relief operation and 11,996 tweets related to evacuation operation.

\subsection{Data Cleaning}

The researchers undergo the data cleaning process using the Waikato Environment for Knowledge Analysis (WEKA) to remove duplicates, URLs, mentions, hashtags, symbols, numbers and upper-case words [12] machine learning tool and notepad++ by means of regular expressions. Only 786 relief-related tweets and 413 evacuation-related tweets are retained after data cleaning.

\subsection{Annotation of Data}

In identifying positive, the researchers manually classified the tweets into positive or negative sentiments. Positive words in English and Filipino in the tweets were identified and analyze to classify the positive sentiments. To identify the negative sentiments, the researchers look for negative words in English and Filipino then analyze the sentiments.

\subsection{Machine Learning}

WEKA was used as machine learning tools for testing the training set; supervised learning was used in training the system to classify the tweets as positive or negative, and stratified 10-fold cross-validation was used to evaluate the classification model results.

The performance assessment is carried out using the standard metrics Accuracy, Precision, Recall and F-Measure to evaluate the training package, as shown in Table 1. 
The dataset is randomly divided into 10 equally sub-sets of approximately equal sizes in 10 -fold cross-validation, and the tweet classes are shown proportionally. The classification model is learned and tested 10 times, the 9- folds being used as the set of training data and the remaining 1 -fold as the set of test data [13]. Likewise, SVM classifier algorithms were used in this study. SVM are two of the most widely used classification algorithms for Machine Learning. SVM is a learning tool that is used in binary grading. The basic concept is to find a hyper plane that divides the d-dimensional data optimally into their two groups [14]. Since the example data is often not linearly separable, SVM implements the notion of a kernel-induced feature space which projects the data into a higher-dimensional space where the data is easier to separate [15].

\begin{tabular}{|c|c|}
\hline Metric & Description \\
\hline Accuracy & $\begin{array}{l}\text { Properly classified sentiments by the } \\
\text { researchers }\end{array}$ \\
\hline Precision & $\begin{array}{l}\text { The researchers' correct positive or } \\
\text { negative feelings about the total amount } \\
\text { of positive or negative feelings the } \\
\text { computer detects. }\end{array}$ \\
\hline Recall & $\begin{array}{l}\text { The number of positive or negative } \\
\text { sentiments identified by the machine } \\
\text { over the total number of manually } \\
\text { classified by the user as positive or } \\
\text { negative sentiments. }\end{array}$ \\
\hline F-Measure & $\begin{array}{l}\text { The precision and recall value multiplied } \\
\text { by two and divided by the sum of } \\
\text { precision and recall value. }\end{array}$ \\
\hline
\end{tabular}

\section{RESULTS AND DISCUSSIONS}

This section presents the data set findings, the preprocessing procedure, the evacuation and relief activity sentiment analysis, and government involvement.

\subsection{Data Set}

From manually classified by the researchers of 13,137 relief operation-related tweets and 11,996 evacuation-related tweets, data cleaning obtains 786 tweets related to relief and 413 tweets related to evacuation with a total of 1,199 sentiment tweets is retained. This was served as the datasets of the study as shown in Table 2.

Table 2: Evacuation and Relief Operation Data Sets

\begin{tabular}{|c|c|c|c|}
\hline Classification & $\begin{array}{c}\text { Positive } \\
\text { Tweets }\end{array}$ & $\begin{array}{c}\text { Negative } \\
\text { Tweets }\end{array}$ & Total \\
\hline Relief Operation & 525 & 261 & 786 \\
\hline Evacuation Operation & 191 & 222 & 413 \\
\hline
\end{tabular}

\subsection{Performance evaluation of the classification model}

The researchers use 10 stratified cross-validations with an algorithm of support vector implemented in machine learning software to test the classification model's performance evaluation. Figure 2 shows the overview results of the 0 -fold stratified cross-validation using SVM as the classifier algorithm for machine learning software to validate the classifier relief process model were released.



Figure 2: Summary Results of Stratified Cross-Validation of Relief Operation Sentiments

The SVM model achieved an average accuracy of 93,0025 percent based on the summary results of stratified cross-validation of relief operation sentiments, indicating that the SVM model categorized 731 unique tweets as being properly classified out of 786 unique tweets. In terms of recall, the SVM classifier classified 521 positive feeling tweets and only 4 positive feeling tweets classified as negative tweets resulting in a 99.2 percent recall value for positive class, while the SVM classifier classified 210 negative feeling tweets for negative class and only 51 negative feelings classified as positive tweets resulting in a recall value of 80.05 percent for negative class. In terms of precision metrics, the sentiment tweets classified $93.4 \%$ of all tweets which the SVM algorithm classifies as positive and F-Measure shows that the classifications of manual tweets are correctly classified with a weighted $92.8 \%$ mean.

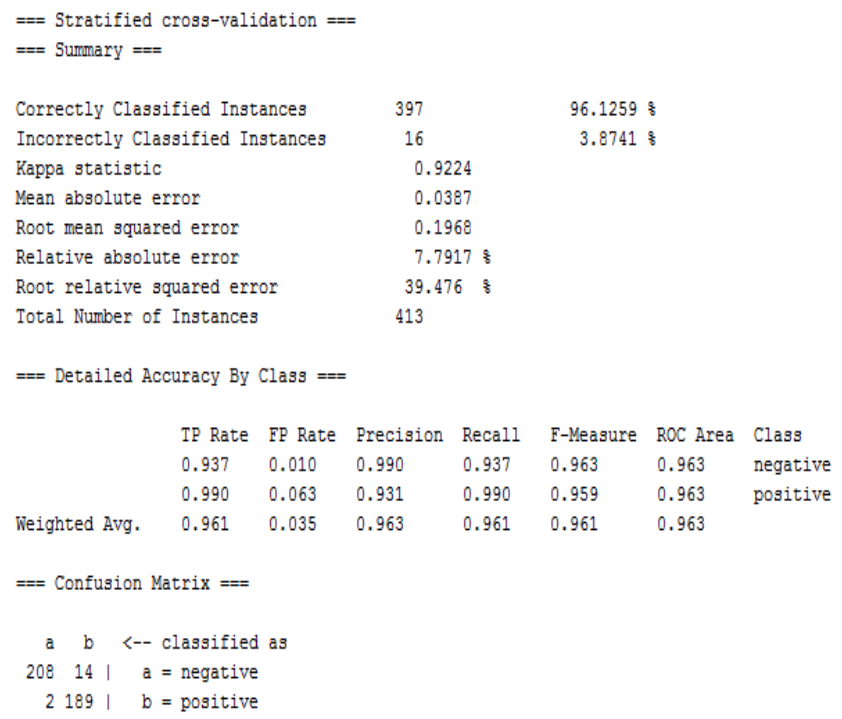

Figure 3: Summary Results of Stratified Cross-Validation of Evacuation Operation Sentiments 
The high percentage rating of the binary categorized data sets received on the True Positive indicated that the manually annotated relief operating data sets were deemed to be right instances. Figure 3 revealed the overview results of the 10 -fold stratified cross validation using SVM as the classification algorithm of machine learning software for validating the evacuation classifier model operation sentiments.

The SVM model achieved an average accuracy of $96.1259 \%$ based on the summary results of stratified cross-validation of evacuation activity feelings, which means that the SVM model categorized 397 unique tweets as being correctly classified out of 413 unique tweets. In terms of recall, the SVM classifier categorized 208 negative sentiment tweets and only 14 negative sentiment tweets classified as positive tweets resulting in a 93.7 percent recall value for negative class while the SVM classifier classified 189 positive sentiment tweets for positive class and only 2 positive sentiments classified as negative tweets resulting in a recall value of $99 \%$. With regard to the precision metric, the SVM algorithm correctly listed the feeling tweets as 96.3 percent of all tweets. And, F-Measure shows that manual tweet classifications are properly classified with a weighted $96.1 \%$ average..

The high percentage rating obtained on the True Negative of the binary classified data sets implied that the manually annotated data sets for evacuation operation were considered to be correct instances.

\subsection{Sentiment Analysis}

Table 3 shows the sentiment tweets for relief and evacuation operations. Based on the manual annotation of the sentiment tweets in relief operation, the sentiment analysis result is $66.79 \%$ positive sentiments, while on SVM Classifier using WEKA, it is $72.77 \%$ positive sentiment tweets on relief operation. It means that the government, non-government organizations and other individuals are supporting one another to have enough reliefs for the victims of the disaster. However, $33.21 \%$ on manual annotation and $27.23 \%$ on SVM Classifier using WEKA has a negative sentiment on relief operations due to the slow process of distribution of reliefs on the affected area.

Table 3: Sentiment Tweets for Relief and Evacuation Operations

\begin{tabular}{|c|c|c|c|c|}
\hline \multirow{2}{*}{ Classification } & \multicolumn{2}{|c|}{$\begin{array}{c}\text { Manual } \\
\text { Annotation }\end{array}$} & \multicolumn{2}{c|}{$\begin{array}{c}\text { SVM Classifier } \\
\text { using WEKA }\end{array}$} \\
\cline { 2 - 5 } & Positive & Negative & Positive & Negative \\
\hline $\begin{array}{c}\text { Relief } \\
\text { Operation }\end{array}$ & $66.79 \%$ & $33.21 \%$ & $72.77 \%$ & $27.23 \%$ \\
\hline $\begin{array}{c}\text { Evacuation } \\
\text { Operation }\end{array}$ & $46.25 \%$ & $53.75 \%$ & $49.16 \%$ & $50.84 \%$ \\
\hline
\end{tabular}

On the other hand, the evacuation sentiment tweets result is $53.75 \%$ having a negative sentiment on evacuation operation on manual annotation process, while in SVM Classifier using
WEKA is $50.85 \%$, suggesting that evacuation process needs some enhancement such as building more evacuation center to avoid the crowded in evacuation. Though, the $46.25 \%$ have positive sentiments tweets on manually annotation and $49.16 \%$ in SVM Classifier using WEKA, since preemptive evacuation process is always done and temporary evacuation is always ready before disaster. It implies that the stratified cross validation of the manual annotation of relief and evacuation sentiment analysis is correctly classified using SVM classifier.

\section{CONCLUSION}

Twitter is one of the subscribers' ways to express their negative or positive opinions or sentiments through their posted tweets about disaster relief and evacuation operations happen in the country before, during and after the disaster. But, as what happened in the data sets of this study, not all tweets are written in English, most of the tweets are written in Filipino languages. In this case, the right pre-processing techniques were used to verify the classification model's performance evaluation. Based on the results of the study, the ratio of positive to negatives tweets related to relief operation is $66.79 \%$ to $33.21 \%$, while on evacuation operation, the ration of negative to positive tweets is $53.75 \%$ to $46.25 \%$. Tweets with positive and negative sentiment tweets will significantly improve the effectiveness and efficiency of relief and evacuation operations.

In addition, 10-fold stratified cross-validation with the application of the SVM algorithm in the machine learning tools will verify the output of the manual classification model even if the data sets are composed of Philippine languages, as long as they are subject to the appropriate pre-processing techniques. The metric of evaluation that could be used to validate the classification model are accuracy, precision, recall, and f-measure.

Thus, research on preprocessing techniques and feature extraction on Filipino languages should be developed using the machine learning tools for an effective and efficient sentiment analysis.

Furthermore, relief operation and evacuation processes are an important procedure before, during and after the disaster. Based on the results, the government needs an intervention to continually provide better relief operations and evacuation process. One of the interventions that the government should take is to enhance the inventory of different resources, specifically foods and non-food items so that relief victims is sufficient on this. Secondly, the government should implement a policy on relief distribution, consider the different factors, such as gender and age issues. And lastly, the government should continue to build a proper, better and safe evacuation center with the proper facilities that the evacuees needed. 


\section{REFERENCES}

1. O. Rodríguez-Espíndola, P. Albores and C. Brewster, Disaster preparedness in humanitarian logistics: a collaborative approach for resource management in floods. European Journal of Operational Research, Vol. 264(3):978-93, 2018. doi: 10.1016/j.ejor.2017.01.021. Available Online at http://www.cbrewster.com/ publications.html; https://ideas.repec.org/a/eee/ ejores/v264y2018i3p978-993.html

2. Z.H. Hu, J.B. Sheu, Y.Q. Yin and C. Wei. Post-disaster relief operations considering psychological costs of waiting for evacuation and relief resources. Transportmetrica A: Transport Science, 13(2):108-3 8, Feb 7, 2017. doi: 10.1080/23249935.2016.1222559. Available Online at https://www.tandfonline.com/ doi/full/10.1080/23249935.2016.1222559

3. A. Nara, X. Yang, S.G. Machiani and M.H. Tsou. An integrated evacuation decision support system framework with social perception analysis and dynamic population estimation. International Journal of Disaster Risk Reduction, 25:190-201, 2017. Available Online at https://par.nsf.gov/servlets/purl/10065918; https://www.nsf.gov/awardsearch/showAward?AWD_I $\mathrm{D}=1634641 \&$ HistoricalAwards=false

4. N. Murzintcev and C. Cheng. Disaster hashtags in social media. International Journal of Geo-Information, 6(7):204, July 5, 2017. doi:10.3390/ijgi6070204. Available Online at https://www.researchgate.net/ publication/318218603_Disaster_Hashtags_in_Social_ Media

5. H. Shekhar and S. Setty. Disaster analysis through tweets. IEEE International Conference in Advances in Computing, Communications and Informatics (ICACCI), pp. 1719-1723, IEEE, August 2015. doi: 10.1109/ICACCI.2015.7275861. Available Online at https://www.researchgate.net/publication/281768488_Di saster_Analysis_Through_Tweets

6. S. K. Yadav. Sentiment analysis and classification: A survey. International Journal of Advance Research in Computer Science and Management Studies, vol. 3(3):113-21, March 2015. Available Online at http://www.ijarcsms.com/docs/paper/volume3/issue3/V3 I3-0032.pdf

7. MonkeyLearn. Sentiment analysis. Jan 2, 2020. Available Online https://monkeylearn.com/sentiment-analysis/

8. S. Al-Asmari and M. Y. Dahab. Sentiment detection, recognition and aspect identification. International Journal of Computer Applications, 177(2):975-8887, November 2017. doi:10.5120/ijca2017915675. Available Online at https://www.researchgate.net/publication/ 322303381_Sentiment_Detection_Recognition_and_As pect_Identification

9. A. Narote, S. Shaikh, S. Pereira, N. Jadhav and P. Rodrigues. Sentiment analysis on Twitter. International Journal of Latest Engineering Research and Applications (IJLERA), ISSN: 2455-7137, Vol. 02, Issue 03, pp. 35-40, March 2017. Available Online at http://www.ijlera.com/papers/v2-i3/4.201703079.pdf
10. J. O. Torio, R. T. Bigueras and D. E. Maligat. Sentiment Analysis on Kto12 Program Implementation in the Philippines. 2018 Fourth International Conference on Advances in Computing, Communication \& Automation (ICACCA), Subang Jaya, Malaysia, 2018, pp. 1-5. doi: 10.1109/ICACCAF.2018.8776852. Available Online at https://ieeexplore.ieee.org/abstract/ document/8776852

11. M. Chapagain. Python: get twitter tweets using 'get old tweets' package. May 8, 2018. Available Online at http://blog.chapagain.com.np/python-get-twitter-tweetsusing-get-old-tweets-package/

12. Machine Learning Group. WEKA: The workbench for machine learning. University of Waikato. Available Online at https://www.cs.waikato.ac.nz/ml/weka/

13. J. Browniee. A Gentle Introduction to k-fold cros-validation. Machine Learning Mastery, May 23, 2018. Available Online at https://machinelearningmastery.com/k-fold-cross-validat ion/

14. R. Nisbet, G. Miner and K. Yale. Handbook of Statistical Analysis and Data Mining Applications, $2^{\text {nd }}$ edition, eBook ISBN: 9780124166455, November 9, 2017. Available Online at https://www.elsevier.com/books/handbook-of-statisticalanalysis-and-data-mining-applications/nisbet/978-0-12-4 16632-5

15. L. P. Lu and N. M. Shara. Reliability analysis: Calculate and Compare Intra- class Correlation Coefficients (ICC) in SAS. Available Online at from https://www.semanticscholar.org/paper/Reliability-analy sis:-Calculate-and-Compare-(ICC)-Lu-Shara/26e8939db e0fa9c9ba8257b4996b840a87e6b455 